\title{
Interseccionalidad: recurso para la producción de espacios urbano-arquitectónicos inclusivos
} Intersectionality: a resource for production of
inclusive urban-architectural spaces

\author{
Karina Contreras Castellanos, ${ }^{\star}$ Eugenio Lara Heyns ${ }^{1}$ y Edgar Fabián Hernández Rivero ${ }^{2}$
}

Fecha de recepción: 15 de enero de 2021

Fecha de aceptación: 11 de marzo de 2021

\begin{abstract}
Resumen - Recientemente, el concepto de interseccionalidad ha adquirido mayor visibilidad debido al interés y a la atención que han despertado diversas luchas y movimientos sociales reivindicativos, entre ellos, y de manera notable, el feminismo. Sin embargo, sus demandas y teorizaciones no parecen haber permeado la consciencia, conocimiento u operación de los/as arquitectos/as y urbanistas en México, así como de otros/as involucrados/as en la producción urbano-arquitectónica. Muchos actos que vulneran, violentan, discriminan o marginan a las personas suceden en el entorno construido; éste, por su parte, no es neutral, sino que desde su materialidad y dinámicas internas, manifiesta y se incorpora a los procesos sociales del contexto, perpetuando o modificando sus constructos. Por ello, consideramos fundamental reflexionar sobre los vínculos entre los planteamientos de los impulsores del cambio social y el quehacer del colectivo relacionado con la producción del entorno edificado. En este artículo, la noción de interseccionalidad tiene una función central, por lo que pone en evidencia el privilegio y el dominio que ciertas condiciones tienen con respecto a otras, y sus posibles cruces, lo cual puede vulnerar a cualquier persona en algún momento.
\end{abstract}

Palabras clave: Interseccionalidad, espacios urbanoarquitectónicos inclusivos, producción urbanoarquitectónica, privilegio-opresión, habitante.
Abstract - Recently, the concept of intersectionality has acquired bigger visibility because of the interest and alert that have awaken in our society a range of social rights fights and movements, among them, and in a remarkable way, feminism. However, their demands and the theories derived from the ideas of these groups do not seem to have passed through the consciousness, knowledge or practice of architects, urbanists, and others involved in the urban-architectural production in Mexico. Most actions that harm, force, discriminate or marginalize people occur in the built environment which, itself, is not neutral. On the contrary, through its materiality and inner dynamics, the built environment manifests -and gets incorporated into- the social processes of the context, perpetuating or modifying its constructs. Because of this, we consider it is fundamental to think about the links between the approaches generated by the ones that drive social change and the collective work related with the production of the built environment. The concept of intersectionality has a main role in this paper for what it makes evident: the privilege and domination that certain conditions have over others -as well as their possible intersections-, which can affect anyone at some point.

Keywords: Intersectionality, inclusive urbanarchitectural spaces, urban-architectural production, privilege-oppression, inhabitant 


\section{Introducción}

I sistema tradicional aún dominante, con sus ámbitos social, político y productivo, se encuentra en crisis y muchas veces sobrepasado para afrontar las problemáticas complejas que se presentan, por ello, es prioritario accionar en distintos niveles para generar cambios profundos. Entre las situaciones que son urgentes de atender se encuentran reducir las desigualdades sociales y respetar los derechos humanos, entre otras razones, para fomentar la integración de la diversidad. Históricamente, los movimientos sociales reivindicativos ${ }^{3}$ han logrado generar grandes transformaciones en torno a estas problemáticas, pues cuando evolucionan se convierten en fuerzas que visibilizan la necesidad de cambios y los exigen. De las diferentes luchas y sus perspectivas se han derivado planteamientos y herramientas que abonan a tener una visión más holística e integral de los asuntos con los que tienen que lidiar. Una de estas nociones es la denominada interseccionalidad.

El concepto de interseccionalidad obtuvo su formalización teórica en 1989, gracias a la exposición que de él hizo la abogada y académica estadounidense Kimberlé Crenshaw, dedicada a defender causas de género y étnicas. Específicamente lo introdujo en la teoría feminista (La Barbera, 2016, p. 106).

Crenshaw identificó y se inspiró en un caso en el que la ley era muy rígida e implicaba diversas variables. Emma Degraffenreid, una mujer afroamericana, demandó a General Motors cuando no la contrataron por dos razones discriminatorias: racismo y sexismo. Degraffenreid argumentaba que, aunque la empresa sí daba trabajo a personas afrodescendientes, se trataba únicamente de hombres destinados a tareas industriales o de mantenimiento, y cuando empleaba a mujeres elegían únicamente a aquellas de piel blanca, con el objetivo de ser recepcionistas o secretarias. Los jueces consideraron el caso inválido al encontrar que presentaba el conflicto de lidiar con distintas variables de discriminación (Crenshaw, 2016).

En dicha situación, Crenshaw pudo visualizar que el problema de los jueces se debía a un error de framing o enfoque, porque éste era parcial y distorsionaba la situación. Para explicar que puede ser más complicado dar solución a una problemática donde se yuxtaponen varias condiciones que tienden a ser discriminadas, la defensora utilizó la siguiente analogía: la demandante, al estar ubicada en la intersección de dos calles, la de género y la racial, recibe un impacto que la deja inconsciente. Una ambulancia (que representa la ley) debe ayudarla, pero según la perspectiva tradicional solo puede hacerlo si ella está sobre una de las calles. Al estar en el cruce de dos calles (sexismo y racismo), la ambulancia no sabe qué hacer y la abandona. A partir de estas ideas Crenshaw desarrolló el denominado feminismo interseccional como un recurso para identificar la superposición o encuentro de factores conflictivos en los problemas a resolver (Crenshaw, 2016).

Otro de los referentes principales a consultar para entender el concepto de interseccionalidad y su sentido es el trabajo de la socióloga y especialista en estudios de género vinculados con la comunidad afroamericana, Patricia H. Collins, quien afirma que la interseccionalidad investiga cómo la confluencia de relaciones de poder influye en las relaciones sociales de las diversas sociedades, así como en las experiencias individuales en la vida cotidiana. Como herramienta analítica, la interseccionalidad observa categorías de raza, clase, género, sexualidad, nación, habilidad, etnicidad y edad -entre otras- que se encuentran interrelacionadas y se moldean mutuamente. La interseccionalidad es una manera de entender y de explicar la complejidad en el mundo, en la gente y en las experiencias humanas (Collins \& Bilge, 2016).

Esto se explica gráficamente con un diagrama que la psicóloga feminista Mary Crawford incluyó

${ }^{3}$ Tales como el feminismo, Black Lives Matter, la lucha por los derechos de la población LGBTQ+, los movimientos ambientalistas o aquellos que defienden el derecho a la vivienda y a la ciudad. 
en su trabajo para representar el significado de interseccionalidad, ${ }^{4}$ a partir de la configuración de un círculo compuesto por ejes que se intersecan al centro mostrando en sus extremos factores que se privilegian en la sociedad contra otros que suelen marginarse, es decir, dicotomías.

\section{Figura 1.}

Diagrama que representa el concepto de interseccionalidad a partir del original incluido en un texto de Crawford (2005).

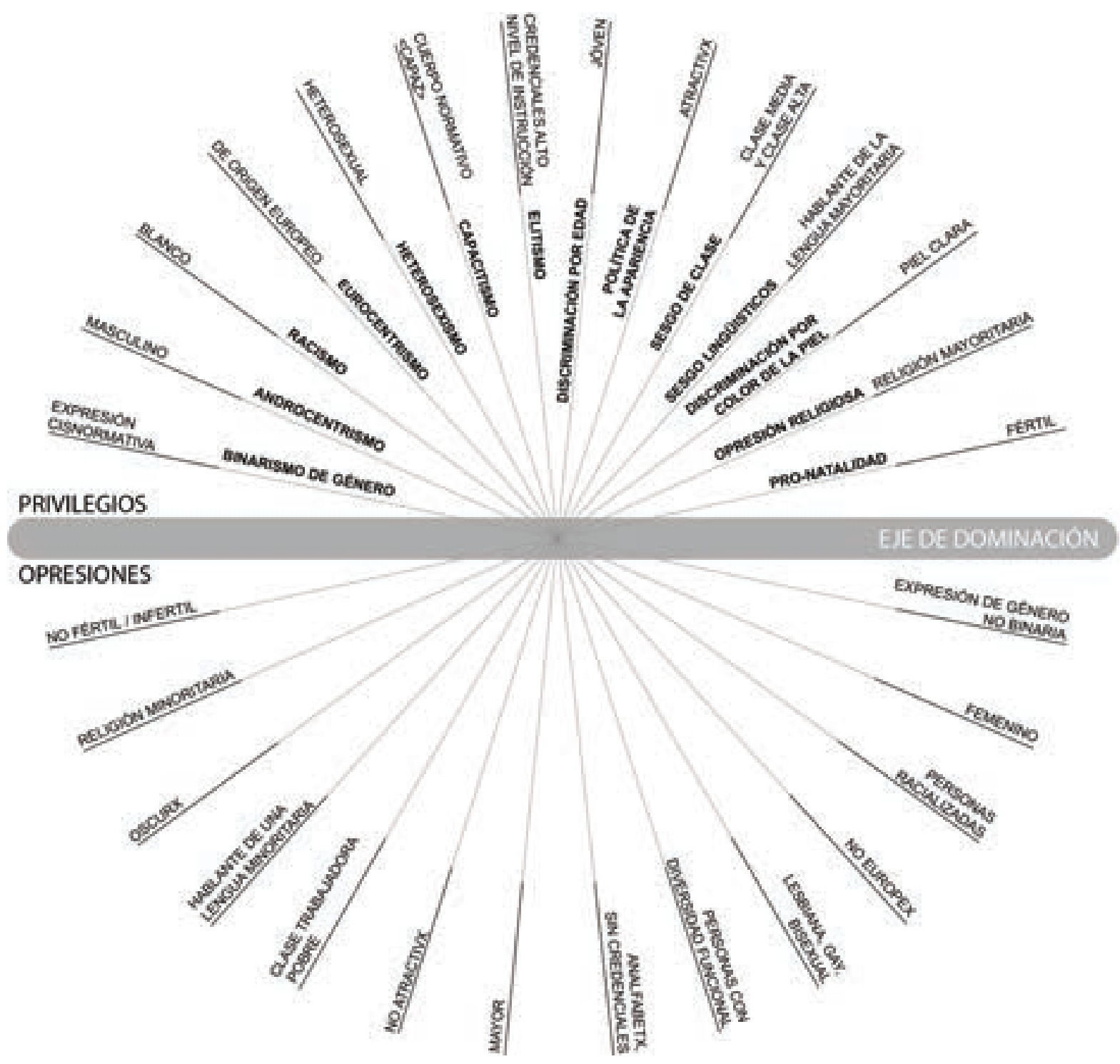

Fuente: Elaboración propia.

\footnotetext{
${ }^{4}$ El término intersección proviene del latín intersectio, compuesto por inter- (entre), secare (cortar), con el sufijo -ción (acción y efecto), dando como resultado su significado: "encuentro entre dos líneas que se cortan recíprocamente" (Etimologías de Chile, s. a.). Esta definición ayuda a entender mejor el gráfico propuesto por Crawford (2005), que representa un círculo configurado por factores de privilegio y de opresión que se cruzan entre ellos.
} 
Este diagrama está compuesto por diversos elementos: la línea horizontal gruesa representa el eje de dominación, llamado por algunos/as autores/as de manera distinta e.g. eje de identidad, que marca la separación o contraposición entre las características o factores que la sociedad privilegia (colocados en la parte superior del esquema) y los que son considerados sus opuestos en cada línea; es decir, los que suelen ser objeto de discriminación u opresión, ubicados en la parte inferior del gráfico. Dependiendo del o la autor/a, el área de privilegios puede adquirir otras denominaciones, tales como eje del Ser o ámbito de la normatividad, mientras que los factores de opresión también se relacionan con el eje del No ser, o con aspectos que desencadenan resistencias sociales. Al plasmar relaciones de tensión y de polarización entre determinadas características de personas o grupos; esta representación evidencia, en última instancia, los prejuicios y las dinámicas de tiranía y dominación que socialmente han sido normalizadas y sus posibles cruces o yuxtaposiciones. Los/as autores/as que han teorizado sobre el concepto de interseccionalidad destacan que su importancia yace en lo que contribuye a lograr como herramienta analítica y no en su definición (Cho et al., 2013 en Collins \& Bilge, 2016). Por lo tanto, un análisis interseccional va más allá de solo hacer uso del término, ya que aporta un marco de referencia para estudiar características, estereotipos y la posible superposición entre ellos, así como formas de relacionarse y conflictos sociales.

A partir de lo que se obtiene con este y otros instrumentos, algunos/as investigadores/as, activistas de la ciudadanía e incluso personas que inciden en las políticas públicas de distintas ciudades alrededor del mundo han podido evaluar contextos sociales, sus problemáticas, la organización de las relaciones de poder en distintos ámbitos, así como diseñar estrategias que son útiles para mejorar condiciones en cada caso de estudio.
Cabe destacar que a diferencia de otras propuestas teóricas y recursos para el análisis de las relaciones sociales, la interseccionalidad, al incluir una amplia gama de aspectos, no se centra únicamente en algunas categorías que hasta ahora han sido las más observadas en este tipo de estudios -como las de género, etnicidad, capacidades físicas y mentales, orientación e identidades, creencias religiosas, estatus migratorio o las que manifiestan el nivel socioeconómico o educativo de las personas-, sino que inserta en el análisis otros elementos que por su sutileza e hipernormalización suelen pasar desapercibidos, tales como la edad, la fertilidad, el estado civil o los parámetros de belleza.

Además de mostrar la amplitud de factores por los que cualquier persona (habitante) puede ser discriminada en algún momento de su vida, observar y atender la realidad desde la óptica de la interseccionalidad muestra que la suma de elementos no privilegiados agrava su situación de vulnerabilidad. Esto es especialmente notorio con la opresión a la que se han visto sometidos, histórica y sistemáticamente, gente y grupos con características o circunstancias que generalmente se menosprecian.

Por lo tanto, es imprescindible que cada uno/a entienda, reflexione, cuestione, señale y demande que estos aspectos sean atendidos y transformados para lograr mayor equidad e integración. Es decir, que se requiere de una ciudadanía activa y crítica, para lo cual es necesario facilitar información y herramientas adecuadas, y promover su importancia para lograr mayor involucramiento y participación colectiva (García \& Ramírez, 2020).

\section{Hacia una incorporación de la interseccionalidad al campo urbano-arquitectónico}

Aunque el concepto de interseccionalidad no sea nuevo y su perspectiva ya se haya empleado para abordar problemáticas del espacio público en algunas 
ciudades europeas y en América Latina ${ }^{5}$ se esté avanzando en su estudio, se ha detectado que en el campo urbano-arquitectónico esta noción es poco conocida, que no existen suficientes fuentes que la relacionen a este ámbito, que su incorporación es incipiente y que cuando se le incluye se ha realizado tangencialmente. Habría que reconocer que recientemente este y otros conceptos han adquirido mayor visibilidad gracias al interés que han despertado el feminismo y la perspectiva de género en el campo, pero aún falta hacer mucho trabajo para que permeen al conocimiento general de arquitectos/as y urbanistas en nuestro país.

Aún es necesario investigar y difundir cómo el contenido, estudio y empleo de la interseccionalidad, como principio y herramienta, puede ser de utilidad para diseñar y evaluar las obras y espacios urbanoarquitectónicos. Hay que apuntar a promover que tanto los/as urbanistas y arquitectos/as, activos/as y en formación, así como otras personas involucradas en la producción del entorno construido, desarrollen una mirada interseccional en su labor.

Para el acercamiento a las maneras como se puede hacer uso de esta perspectiva en el ámbito urbanoarquitectónico habría que señalar, en primer lugar, que en la producción, percepción e interpretación del entorno construido se agregan y manifiestan constructos socioculturales de los distintos grupos en los que conviven los individuos. Posteriormente, se debe entender que los espacios, a su vez, influyen en las interacciones sociales y en la experiencia personal, lo cual resulta en el entendimiento que los/as usuarios/as tienen de esos sitios. $Y$ es que aunque los entornos urbano-arquitectónicos y su historia no determinan el comportamiento y la vida de los seres humanos, varios/as investigadores/as han demostrado que las características de las obras y los espacios, junto con factores contextuales, sí tienen efectos en diversos grados en las personas y en las relaciones sociales. ${ }^{6}$ Entre estos efectos se encuentra la interpretación que un usuario realiza de un espacio que experimenta, lo cual se asocia a que en un sitio se sienta integrado o, por el contrario, excluido. Con base en lo anterior se podría hablar de que existen lugares urbanoarquitectónicos inclusivos y, en contraposición, otros de exclusión en los que no se fomenta la incorporación de la diversidad social.

Pero, ¿qué es un espacio inclusivo? Se puede entender como un lugar donde toda la gente se siente bienvenida por igual y en el que se facilita la convivencia armónica y respetuosa, sin que sus distintas características o perspectivas sean impedimento para ello. Además, es importante entender que un espacio inclusivo, considerando otras cualidades, también debería ser habitable en correspondencia con los requerimientos de sus distintos/as usuarios/as. Recíprocamente, la inclusión es uno de los factores para lograr la habitabilidad.

Así pues, en lo urbano-arquitectónico, inclusión e interseccionalidad están correlacionadas, ya que alcanzar genuinamente la primera en los espacios requiere de una mirada interseccional que permita identificar cómo las cualidades físicas de los sitios, existentes o a diseñar, se vinculan con las relaciones humanas y con el cruce de factores o características que en ellas y en las personas se asocian con el privilegio o el menoscabo. Sin embargo, es imprescindible comprender que la mejora de los entornos compartidos es un proceso complejo, de manera que conseguirlo no sólo depende de urbanistas, arquitectos y profesionales de otras disciplinas implicados en la producción urbano-arquitectónica, sino que, tal como

${ }^{5}$ Ciudades europeas como Barcelona y Berlín son ejemplo de cómo se ha integrado de manera efectiva la perspectiva de género, incluyendo la interseccionalidad y la transversalidad en sus estrategias y políticas públicas para la planeación urbana y así mejorar los espacios compartidos por sus habitantes. En América Latina, países como Argentina y Chile encabezan el trabajo transdisciplinario en torno a estas cuestiones.

${ }^{6}$ Investigadores [e.g. Bagley (1974), Taylor (2002), Gómez-Azpeitia, Cruz, Gómez \& Alcántara (2005)] de distintos ámbitos disciplinares como la sociología, la psicología ambiental y la arquitectura, entre otras, han hecho estudios que confirman la influencia que tienen los espacios en el comportamiento de las personas a nivel individual y colectivo. 
lo indican Hancock (2020) y otros/as (García \& Ramírez, 2020; UN-HABITAT, 2020), es fundamental contar con políticas públicas adecuadas y la participación crítica de la sociedad civil, ya que sus miembros expresan sus necesidades $y$, en última instancia, habitan y validan el espacio. Es así como el surgimiento de lugares inclusivos inevitablemente demanda la colaboración activa de sociedad, profesionales y gobierno.

\section{Figura 2.}

Intersección de las esferas que intervienen en la configuración y transformación del entorno construido: la sociedad, el ámbito gubernamental y el colectivo encargado de la producción urbano-arquitectónica (planeación, diseño y construcción).

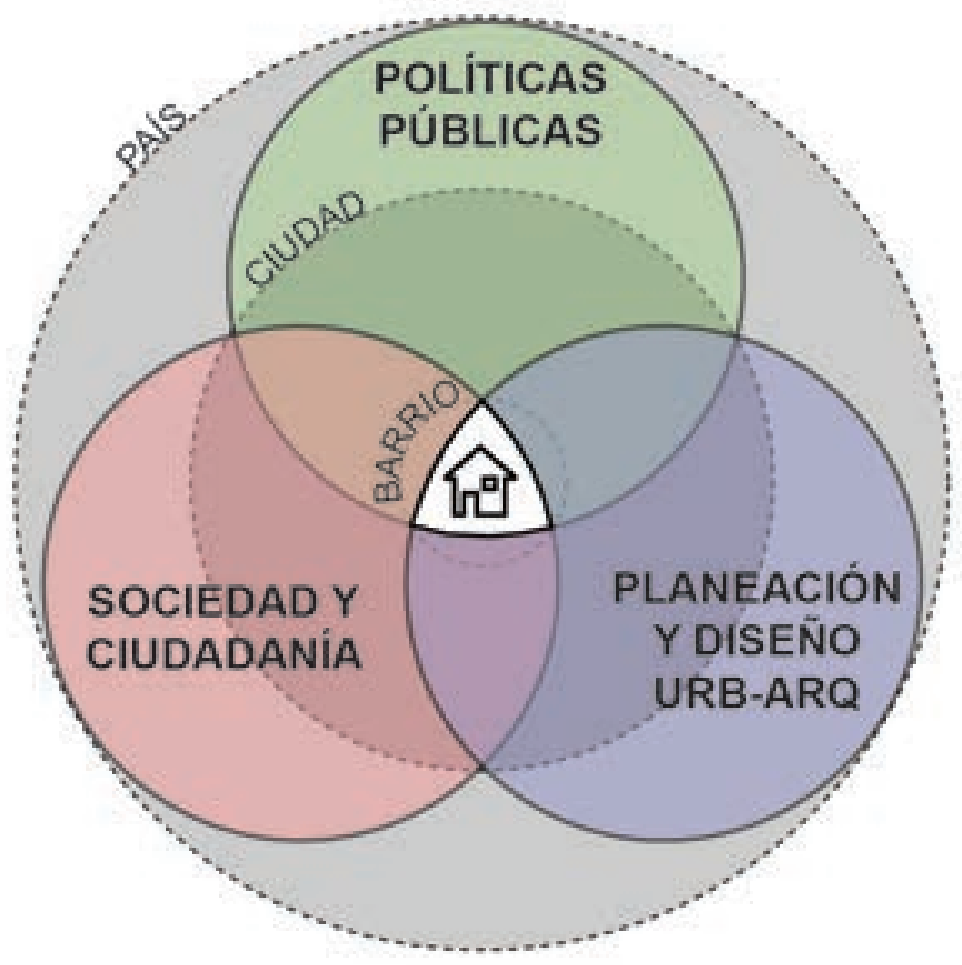

Fuente: Elaboración propia.

\section{Espacios de exclusión vs. espacios inclusivos}

La relación inclusión/exclusión puede abordarse en distintas escalas del entorno construido y abarcar desde la ciudad, el barrio o incluso la vivienda. Sin embargo, la observación de estos fenómenos se facilita en los espacios compartidos, interiores o exteriores, con carácter público o semipúblico: calles, plazas, parques, estaciones de transporte, escuelas, oficinas, centros comerciales, mercados o zonas comunes en conjuntos habitacionales. $Y$ es que ahí es donde se espera que se susciten más cruces e interacciones entre los diferentes habitantes, con sus divergencias y sus coincidencias, aunque en muchos casos no sucede porque son sitios inhóspitos, por lo que es esencial pensar que en estos ambientes se estimule una convivencia armónica y segura.

La oposición entre la exclusión y la inclusión surge de la dicotomía que se ha establecido en el sistema dominante entre los factores privilegiados 
y los marginados. Este antagonismo se manifiesta en el entorno construido, porque éste es producido dentro del marco social. Al respecto, las feministas señalan que esto es consecuencia de que las grandes urbes contemporáneas se hayan concebido desde un punto de vista capitalista y patriarcal. Lo anterior se asocia, entre otras cosas, con relaciones de inclusión/exclusión entre lo masculino y lo femenino en los espacios, por ejemplo, en lugares públicos se tiende a favorecer lo masculino, mientras que lo femenino, y con ello las labores domésticas vinculadas a este género, es relegado al espacio privado (García \& Ramírez, 2020).

Desde una mirada interseccional, la utilización personal del espacio se disfruta o se padece en función del nivel de privilegio con el que se cuente, no sólo por los preconceptos sociales, sino por la manera en que los entornos se diseñan y materializan, pues éstos promueven a su vez ciertas dinámicas sociales. Pero la segregación o integración atribuible a los espacios no sólo depende de su percepción como objetos aislados, sino también de la asimilación de estas materialidades en conjunto con varios elementos de la pluralidad del contexto, que van desde los aspectos naturales y físicos, hasta los acontecimientos y las relaciones suscitadas entre los diferentes objetos y seres presentes. De todo ello, el/ la usuario/a hace una síntesis, captando atmósferas complejas que cambian a lo largo del tiempo.

No obstante, aunque la evaluación que realizan los/las usuarios/as de los espacios que utilizan y experimentan no resulta exclusivamente de los estímulos percibidos acerca de sus cualidades físicas, los/as arquitectos/as y diseñadores/ as urbanos/as deberían tener el conocimiento y la responsabilidad para identificar y establecer conexiones entre las características de los espacios, los factores intangibles del entorno y las repercusiones que la suma de esto tiene en las personas, y ser capaces de integrar esta información en la toma de decisiones. Los/las profesionales de la creación de espacios tendrían que ser conscientes de que las características físicas de lo que conciben, en vinculación con su contexto, pueden ser leídas o significadas por los seres humanos combinando diferentes tipos de asociaciones, las cuales se pueden clasificar en tres categorías: las que apelan a la psicobiología que compartimos como especie en general, que aun con ello presentan particularidades (edad, capacidades y estado de salud, sexo, etnicidad, etcétera), las que corresponden a los códigos socioculturales de cada comunidad donde se desenvuelven los individuos (el género como constructo social, situación socioeconómica, identidad cultural, entre otras) y otra categoría que deriva directamente de su experiencia personal, pero que está siempre relacionada con las dos primeras (Contreras, 2020, p. 207-210).

Además de que la interpretación positiva o negativa del/la usuario/a del entorno construido tiene que ver con sus características personales y con las distintas asociaciones que establece según sus códigos, también esta evaluación depende de su rol en los espacios y todo ello se corresponde con el tipo de actividad que desempeña en determinado momento. De esta manera, y tomando en cuenta que la experiencia del entorno urbano-arquitectónico puede quedar a nivel inconsciente y con una evaluación preintelectual, existen propiedades o sensaciones derivadas de su percepción que pueden pasar desapercibidas para mucha gente y solo cobran importancia para aquellos/as a quienes afectan positiva o negativamente de manera muy palpable. Por ejemplo, un muro muy largo, alto y ciego junto a una banqueta estrecha al lado de una calle vehicular transitada puede ser asociado por un peatón como límite físico y factor que provoca inseguridad, mientras que el/la automovilista no repara en ello y centra su atención en la fluidez del tráfico de la calle.

Es indiscutible que, en gran parte de las ciudades, al priorizarse al o a la automovilista, el peatón o la 
peatona suele enfrentar más desventajas, pero si además se yuxtaponen otras condiciones como que el/la transeúnte sea un/a adulto/a mayor, o que tenga que utilizar una silla de ruedas, la situación se vuelve más adversa para su movilidad y percepción.
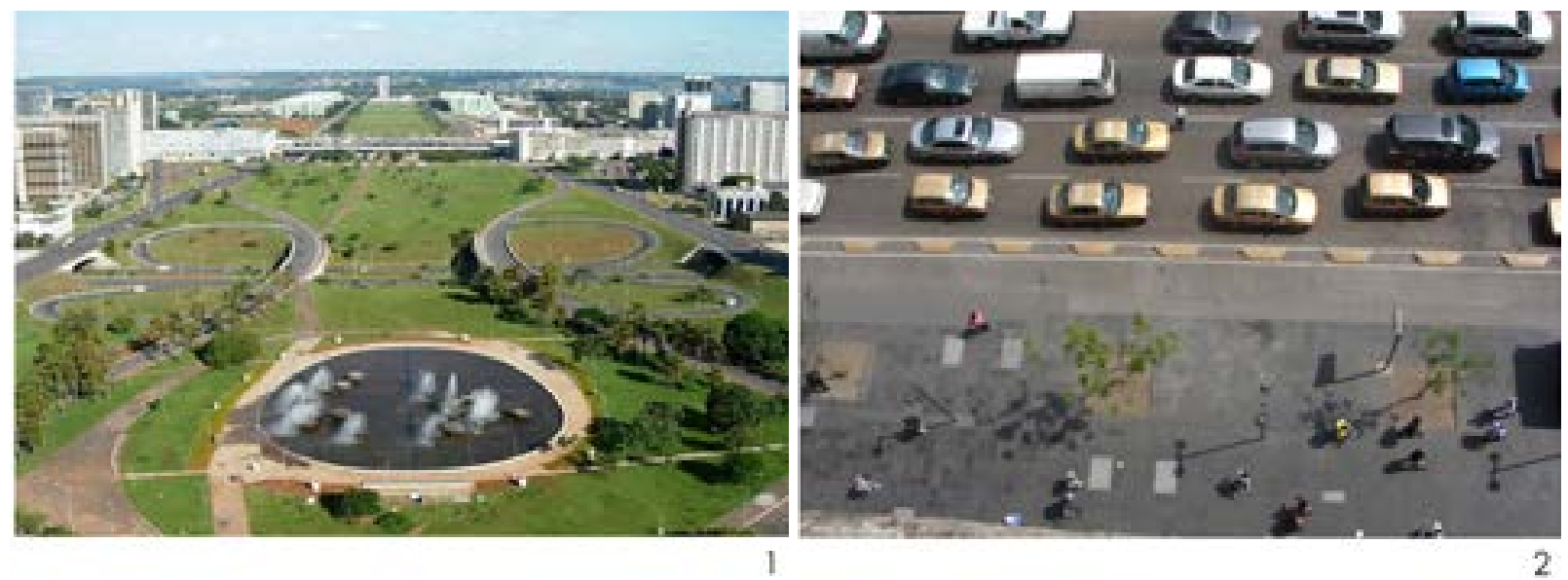

Las ideas de progreso y del capitalismo, absorbidas en los ideales del urbanismo moderno, originaron el privilegio del/de la automovilista por encima del peatón o peatona. Esto se normalizó a tal grado que sólo recientemente se ha empezado a cuestionar: Brasilia, ciudad moderna vs. las intervenciones que, considerando distintas movilidades, se realizan recientemente en sitios como Ciudad de México. Izquierda: Brasilia, Brasil. Fotografía de Karina Contreras, 2006. Derecha: Centro Histórico, Ciudad de México. Fotografía dé Édgar Hernández, 2015.

Otro ejemplo de cómo el diseño y la planeación urbana favorecen la exclusión sucede en varios de los centros históricos de las ciudades mexicanas, pues éstos tienden a gentrificarse para convertirlos prioritariamente en lugares turísticos y de consumo, dejando de lado su potencial habitable y disponibilidad para todos/as. En ciudades como Oaxaca o San Miguel de Allende se promueve o facilitan prácticas que derivan en el cambio de uso de suelo y en el incremento del costo de vida para los/as habitantes originales, lo que eventualmente motiva su expulsión. Con este tipo de acciones gentrificadoras también se refuerzan la discriminación y la marginación de otros integrantes de la población, como sucede con los/as indígenas, pues ellos/as quedan casi siempre relegados/as a formar parte de la atmósfera turística y folclórica, por lo que su actividad en estos sitios se limita generalmente a lo comercial; pero aunque puedan vender sus productos, el problema reside en que no cuentan con las mismas prerrogativas que tienen los/as mestizos/as o los/as extranjeros/as para moverse, utilizar y disfrutar de los lugares compartidos. Es decir, que en estos y en muchos otros sitios, aunque se hable del derecho a la ciudad, ${ }^{7}$ en la práctica la ciudad no es para todos/as.

Estas situaciones son consecuencia tanto del sistema económico vigente -incluyendo las relaciones de poder que lo sustentan y las políticas que le dan continuidad- como de las ideas y prejuicios de la sociedad en general. Estas fuerzas, a su vez, suelen marcar pautas para la planeación y la producción urbanoarquitectónica, de manera que el entorno construido es y expone mucho de lo que somos como sociedad, asunto que es sumamente necesario cuestionar, reflexionar y modificar cuando se requiera.

\footnotetext{
${ }^{7}$ El derecho a la ciudad es un concepto desarrollado a mediados del siglo XX por Henri Lefebvre, en el que se alude al pleno acceso y disfrute del entorno urbano. En términos de diseño y puesta en práctica de políticas públicas urbanas, se puede referir a que todos/as los/as habitantes tienen derecho a una vivienda asequible, trabajo y salario dignos, alimentos frescos y sanos, educación, salud, así como lugares seguros y libres de contaminación, según el punto de vista planteado por Colau \& Font (2020).
} 


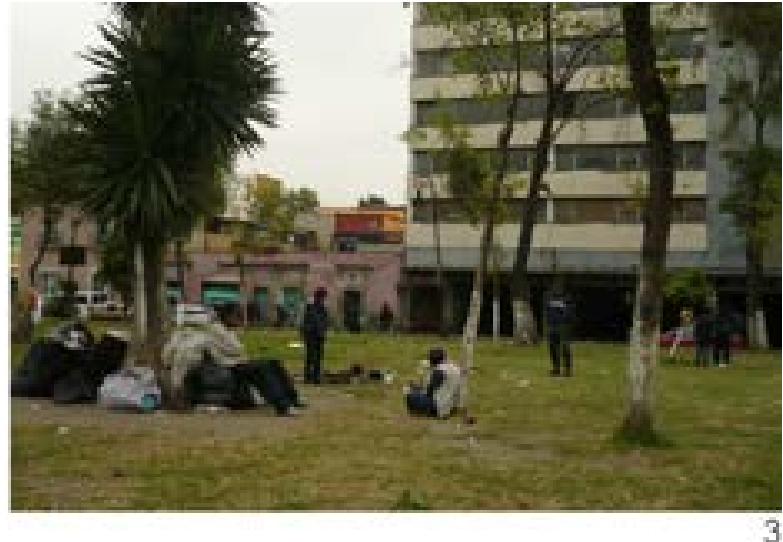

Plaza de la Soledad, Merced Balbuena, Ciudad de México. Fotografía de Eugenio Lara Heyns, 2013.

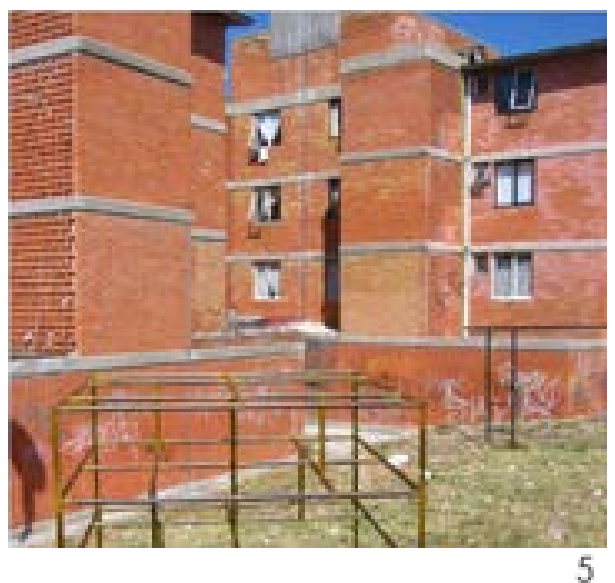

Infonavit La Margarita, Puebla, Pue.

Fotografía de Eugenio Lara Heyns, 2008.

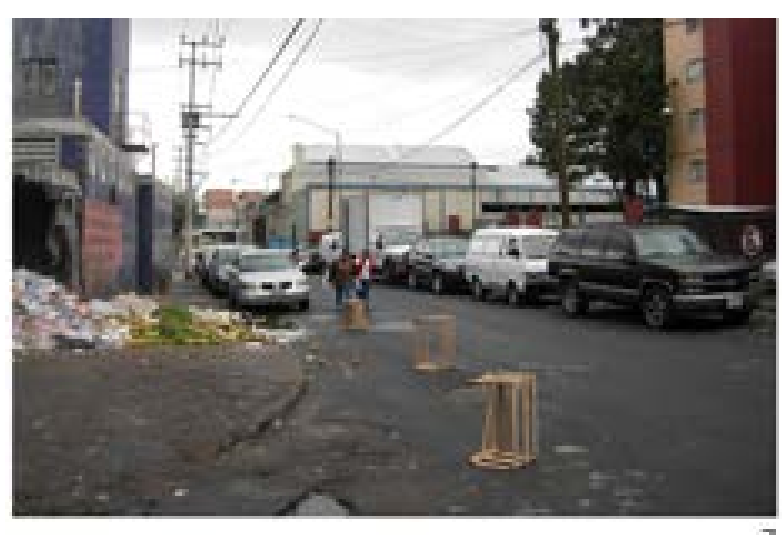

Merced Balbuena, Ciudad de México.

Fotografía de Karina Contreras, 2013.

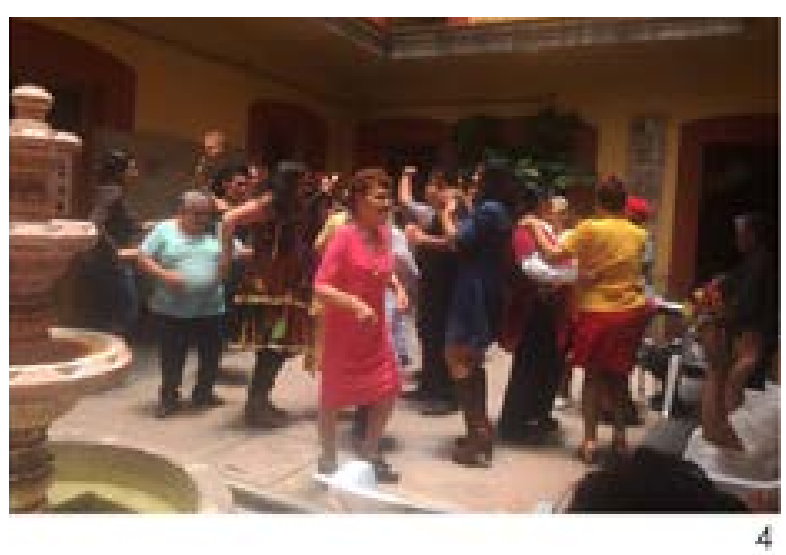

Casa Xochiquetzal, Centro, Ciudad de México.

Fotografía de Karina Contreras, 2017.

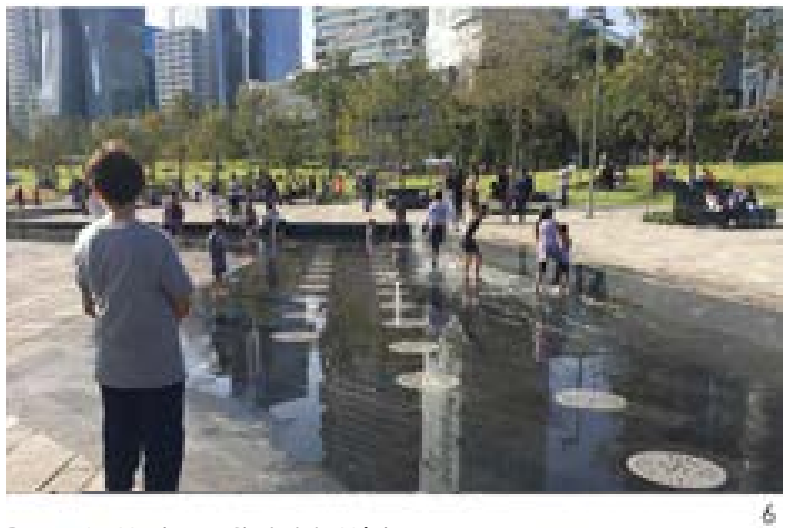

Parque La Mexicana, Ciudad de México.

Fotografía de Karina Contreras, 2018.

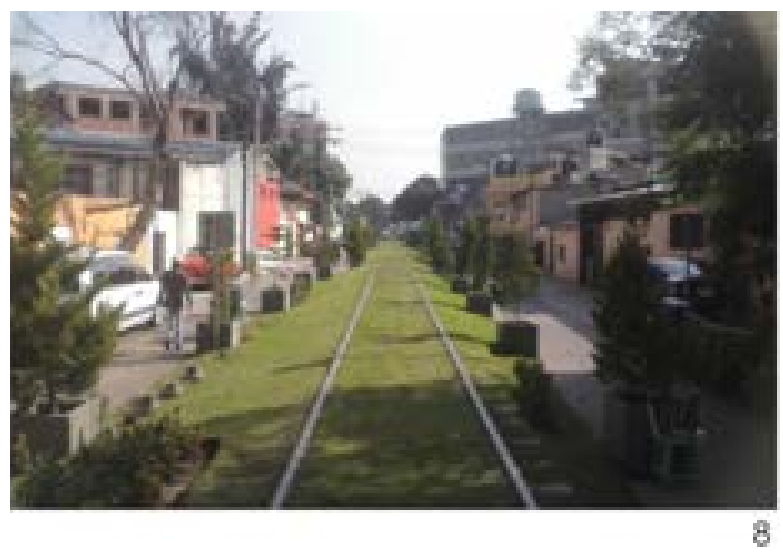

Tramo del FFCC de Cuernavaca, Granada, Ciudad de México. Fotografía de Édgar Hernández, 2015.

Ejemplos de espacios que excluyen y segregan frente a espacios inclusivos. Fotos 3-4: Sitio degradado utilizado por indigentes y adictos vs. Casa Xochiquetzal, espacio de reivindicación para trabajadoras sexuales adultas mayores. Fotos 5-6: espacio de juego en desuso vs. espacio lúdico que propicia la convivencia. Fotos 7-8: un entorno degradado genera un círculo vicioso de descuido, abandono e inseguridad vs. un espacio cuidado favorece la apropiación colectiva de sus habitantes y se percibe como más seguro. 
Visto de manera general, hay algunos factores fundamentales a tomar en cuenta que resultan en una interpretación negativa o positiva de los espacios y de su nivel de inclusión: la seguridad percibida, el grado de accesibilidad y movilidad que facilitan y la convivencia armónica y pacífica que entre habitantes y su diversidad se posibilita. En los lugares de exclusión, la inseguridad que se percibe a partir de los atributos y del estado físico del entorno, en combinación con factores sociales, genera la sensación de miedo, emoción que alerta para la sobrevivencia. La inseguridad y el miedo se asocian con la ausencia de equipamiento adecuado en los espacios o con su degradación: la falta de luz y vegetación, la presencia de basura y el descuido excesivo de lo material o su abandono, generalmente son componentes que se correlacionan con sitios en los que se desarrollan conflictos sociales, lo cual incluye la violencia y la criminalidad. Si, en cambio, se favorece la apropiación espacial ${ }^{8}$ de los habitantes, se propicia el mantenimiento físico y social del entorno, lo que contribuye a connotarlo como seguro.

El rol del miedo en las ciudades y en los lugares determina cómo todos los experimentamos, pues esta sensación afecta las decisiones tomadas para poder sobrellevar la vida cotidiana en el medio construido. No obstante, existen grupos más vulnerables porque pueden tener mayor afectación, tal como sucede con las mujeres y los/as niños/as.

Por su parte, una buena convivencia entre los/as habitantes se relaciona tanto con la seguridad, pues un espacio vivo se interpreta como menos inseguro, como con una accesibilidad y posibilidad de movilidad en el lugar adecuado, para que todos/as los/as usuarios/as, sin importar edad, capacidades físicas o psicológicas, clase social, género, orientación sexual, etnia, ideología, etcétera, puedan converger.
Estos tres factores interrelacionados -seguridad, accesibilidad/movilidad y la estimulación de una convivencia apropiada- se ven favorecidos no únicamente con la existencia de equipamiento suficiente, con el buen mantenimiento de los espacios o con un diseño que evite barreras físicas considerando las distintas capacidades de las personas por salud o por edad, también es fundamental complementar estas acciones incorporando la naturaleza, por sus efectos restaurativos físicos y psicológicos (Collado, Staats, Corraliza \& Hartig, 2017). Asimismo, es necesario fomentar la diversidad de usos, horarios y personas en el sitio y en su entorno próximo (Colau \& Font, 2020; García \& Ramírez, 2020; Muxí, 2020a).

Urbanistas y arquitectos/as, como otras personas involucradas en la planeación y el diseño del espacio, requieren comprender mejor las problemáticas que enfrentan, así como indagar e integrar conocimientos y herramientas para mejorar el entorno construido y su impacto en el contexto sociocultural, políticoeconómico y natural. Por lo tanto, es fundamental considerar los cruces y las yuxtaposiciones entre privilegios y aspectos de opresión que se vinculan con las características de los/as habitantes y con sus relaciones sociales. Aquí es donde específicamente la interseccionalidad puede encontrar su lugar para incorporarse como un recurso útil en la planeación y la materialización de espacios más inclusivos y solidarios.

\section{La interseccionalidad como recurso a integrar en la creación de espacios inclusivos}

Como se ha expuesto, la interseccionalidad es principalmente una herramienta analítica; por lo tanto, es así como se puede incluir en los procesos de planeación urbana y diseño de espacios, contribuyendo a visibilizar información y aspectos

\footnotetext{
${ }^{8}$ La apropiación espacial puede ser entendida como la adaptación activa y positiva que tienen los/as usuarios/as a las obras y los espacios urbanoarquitectónicos con calidad habitable. Es lo opuesto a una adaptación pasiva, donde las personas se subordinan a los espacios y muchas veces los padecen (Contreras, 2020).
} 
con los cuales trabajar. Ahora bien, no se pueden enunciar estrategias estandarizadas para propiciar una mirada interseccional en la evaluación y producción urbanoarquitectónica, debido a que cada caso a abordar es único. Sin embargo, es posible esbozar planteamientos generales a considerar.

Para comprender cómo sumar la interseccionalidad a la concepción y materialización de espacios inclusivos, es pertinente revisar cómo se ha hecho hasta ahora, junto con la perspectiva de género y los objetivos para fomentar la inclusión en programas diseñados por miembros de las esferas gubernamentales y no gubernamentales de diferentes partes del mundo. El resultado de este ejercicio puede utilizarse como modelo a ser adaptado y desarrollado para abordar los distintos problemas de planeación y diseño urbano-arquitectónico a resolver acorde con sus particularidades. Algunos ejemplos de medidas que ya se han añadido a las políticas públicas en diversos países son las que han dado lugar a agregar la perspectiva de género en la transversalización del desarrollo urbano (gender in mainstreaming urban development) de la ciudad de Berlín (Droste, 2011); las iniciativas que provienen de los programas de las Naciones Unidas para los Asentamientos Humanos (UNHABITAT), con el fin de promover al espacio público como una pieza clave para instaurar ciudades sustentables que brinden buena calidad de vida para toda la población;' las estrategias que se han puesto en práctica con base en el concepto de ciudad cuidadora ${ }^{10}$ en Barcelona, donde en el gobierno, además, se han formado consejos de mujeres desde 1994 que han evolucionado hasta llegar a conformar un departamento de Feminismo y LGBT, así como de un Plan para la Justicia de Género (Hancock, 2020); o las acciones que se han propuesto en Chile para la planeación urbana de ciudades sanas, seguras, sustentables y solidarias (Gehl, 2017).
A las antes mencionadas se pueden adicionar los proyectos ciudadanos que también impulsan cambios en las políticas o que se han originado de ellas, tal como las del Col.lectiu Punt 6 de Barcelona, que se fundó en 2004 a partir de la formalización de la Ley de Barrios, o el colectivo argentino Ciudad del Deseo, nacido como resultado del Paro Internacional de Mujeres 2019 en Buenos Aires. Aquí también cabe mencionar planteamientos como los que buscaban la mejora de viviendas y el uso de lugares abandonados para su ocupación temporal con fines sociales y basado en el trabajo colectivo realizado por asociaciones de mujeres en Chicago, entre el siglo XIX y el XX (Muxí, 2020b).

Desde la esfera de la planeación y el diseño urbanoarquitectónico no sólo se interviene en la configuración de las propuestas que se incorporan al ámbito político, también se han generado ideas y hechos más específicos. Esto implica planteamientos para la desgenerización de los espacios doméstico y privado, y de las labores tradicionalmente asociados a ellos y a la dicotomía entre lo masculino y lo femenino, como las que ha emprendido la arquitecta Zaida Muxí con distintos/as colaboradores/ as, entre ellas "Walking India: Women Resource Zone", realizado entre 2010 y 2013 con la participación de Shivani Bhardwaj y la organización Equal Saree, con el objetivo de crear redes seguras para la vida cotidiana de mujeres en diferentes ciudades (Equal Saree, 2016); o incluso las que se formularon en otras épocas, como las intervenciones para acondicionar espacios de juegos para niños/as de Jakoba Mulder y Aldo Van Eyck hacia la segunda mitad del siglo pasado (Muxí, 2020b).

En el Cuadro 1 se sintetizan los puntos más relevantes contenidos en varias estrategias formuladas en cada una de las distintas esferas y sus sinergias: gubernamental, ciudadana y la de la planeación y diseño urbano-arquitectónico.

${ }^{9}$ En la Ciudad de México, por ejemplo, se han contemplado ideas para políticas basadas en las propuestas de la ONU orientadas a crear ciudades seguras para las mujeres y las niñas, desde 2016 (Hancock, 2020).

${ }_{10}$ Se refiere a que la ciudad debe cuidar a sus habitantes facilitando su autonomía y suministrando lo necesario para una vida digna, incluyendo la vivienda, la salud, la alimentación, cuidado medioambiental e incluso apoyo para que las personas no se sientan solas. Es una aportación del urbanismo feminista que marca un giro con el paradigma anterior, basado en el urbanismo androcéntrico. 


\section{Cuadro 1.}

Algunas estrategias y planteamientos con perspectiva de género e interseccionalidad para la generación de espacios inclusivos y solidarios

\begin{tabular}{|c|c|c|c|}
\hline $\begin{array}{l}\text { INDIVIDUOS, GRUPOS } \\
\text { Y PLANTEAMIENTOS } \\
\text { IMPULSORES DE CAMBIOS }\end{array}$ & $\begin{array}{l}\text { ESFERA: POLÍTICA/ } \\
\text { CIUDADANA/ } \\
\text { EXPERTOS }\end{array}$ & $\begin{array}{l}\text { LO QUE SE ENFOCA } \\
\text { DE LA PROPUESTA }\end{array}$ & $\begin{array}{l}\text { ASPECTOS A DESTACAR EN LAS } \\
\text { ESTRATEGIAS QUE PROPONEN }\end{array}$ \\
\hline \multirow{2}{*}{$\begin{array}{l}\text { The Public Space Site-specific } \\
\text { Assessment. Guidelines to } \\
\text { achieve quality public spaces } \\
\text { at neighbourhood level. } \\
\text { (UN-HABITAT, 2020) }\end{array}$} & \multirow{2}{*}{$\begin{array}{l}\text { Organización internacional } \\
\text { POLÍTICA }\end{array}$} & Fases & $\begin{array}{l}\text { 1. Preevaluación con investigación } \\
\text { documental de cada caso. 2. Recopilación de } \\
\text { datos con distintos métodos participativos. } \\
\text { 3. Análisis. } 4 \text {. Seguimiento y evaluación de la } \\
\text { implementación }\end{array}$ \\
\hline & & Dimensiones & $\begin{array}{l}\text { 1. Uso y usuario. 2. Accesibilidad. } \\
\text { 3. Amenidades y mobiliario urbano. } \\
\text { 4. Confort y seguridad. } 5 \text {. Integración de } \\
\text { ambientes verdes. }\end{array}$ \\
\hline $\begin{array}{l}\text { Ciudad del Deseo, Argentina } \\
\text { (Maiztegui, 2020) }\end{array}$ & $\begin{array}{l}\text { Colectivo feminista - } \\
\text { CIUDADANA }\end{array}$ & Recomendaciones & $\begin{array}{l}\text { 1. Construcción de equipamiento urbano } \\
\text { de barrios pensado desde la accesibilidad. } \\
\text { 2. Replantear un sistema de transporte } \\
\text { que favorezca la multiplicidad de viajes y } \\
\text { accesibilidad y seguridad para quienes son } \\
\text { cuidados. 3. Hacer ciudad desde la óptica } \\
\text { feminista. }\end{array}$ \\
\hline $\begin{array}{l}\text { Christie Greiner } \\
\text { (Arellano, 2020) }\end{array}$ & $\begin{array}{l}\text { Profesora de Comunicación y } \\
\text { Artes: Pontificia Universidad } \\
\text { Católica de Sao Paulo - } \\
\text { EXPERTOS }\end{array}$ & $\begin{array}{l}\text { Puntos en común que } \\
\text { relacionan los cuerpos } \\
\text { individuales }\end{array}$ & $\begin{array}{l}\text { 1. Dislocamientos espacio-temporales. } \\
\text { 2. Transiciones desde lo privado-individual } \\
\text { a lo público-colectivo 3. Alianza entre } \\
\text { naturaleza y cultura. } 4 \text {. Ambivalencia entre } \\
\text { inestabilidad y estabilidad. 5. Política de las } \\
\text { acciones y su visibilidad. }\end{array}$ \\
\hline $\begin{array}{c}\text { Ada Colau } \\
\text { (Colau, 2020) }\end{array}$ & $\begin{array}{l}\text { Alcaldesa de Barcelona - } \\
\text { POLIITICA }\end{array}$ & $\begin{array}{l}\text { Ciudad Cuidadora. } \\
\text { Características }\end{array}$ & $\begin{array}{l}\text { 1. Desarrollo de la educación. 2. Provisión } \\
\text { de alimentos sanos y frescos. 3. Contemplar } \\
\text { el derecho a la salud y gozo de los espacios } \\
\text { libres de contaminación. } 4 \text {. Vivienda digna. } \\
5 \text {. Cuidado de personas que se sientan } \\
\text { desamparadas. }\end{array}$ \\
\hline $\begin{array}{c}\text { Nueva Agenda Urbana } 2030 \text { (ONU- } \\
\text { HABITAT, 2017) }\end{array}$ & $\begin{array}{l}\text { Organización internacional } \\
\text { POLIITICA }\end{array}$ & $\begin{array}{l}\text { Objetivo Desarrollo } \\
\text { Sustentable no. } 11\end{array}$ & $\begin{array}{l}\text { "Hacer ciudades y asentamientos } \\
\text { humanos inclusivos, seguros, resilientes y } \\
\text { sustentables" }\end{array}$ \\
\hline \multirow{3}{*}{$\begin{array}{l}\text { La dimensión humana en el espacio } \\
\text { público, recomendaciones para el } \\
\text { análisis y el diseño } \\
\text { (Gehl, 2017) }\end{array}$} & \multirow{3}{*}{$\begin{array}{l}\text { Ministerio de Vivienda } \\
\text { y Urbanismo de Chile + } \\
\text { ONU + Gehl Architect - } \\
\text { POLIITICA + EXPERTOS }\end{array}$} & $\begin{array}{l}\text { Ciudades hechas a medida } \\
\text { de quién las habita. } \\
\text { Características }\end{array}$ & $\begin{array}{l}\text { 1. Seguras. 2. Sustentables. 3. Solidarias. } \\
\text { 4. Vitales. }\end{array}$ \\
\hline & & Fases de trabajo & $\begin{array}{l}\text { 1. Investigar. 2. Envisionar. 3. Estrategar. } \\
\text { 4. Diseñar. 5. Implementar. 6. Evaluar. }\end{array}$ \\
\hline & & Ámbitos de actuación & $\begin{array}{l}\text { 1. Preservación del patrimonio. 2. Movilidad } \\
\text { sustentable. 3. Equidad y diversidad. } \\
\text { 4. Diseño urbano a escala humana } 5 . \\
\text { Fomento económico y cultural }\end{array}$ \\
\hline $\begin{array}{l}\text { Col.lectiu Punt 6, Barcelona } \\
\text { (Col.lectiu Punt 6, 2016) }\end{array}$ & $\begin{array}{l}\text { Colectivo feminista - } \\
\text { CIUDADANA }\end{array}$ & Recomendaciones & $\begin{array}{l}\text { 1. Proximidad 2. Diversidad de usos } 3 . \\
\text { Autonomía (accesibilidad y seguridad). } \\
\text { 4. Vitalidad. 5. Representatividad }\end{array}$ \\
\hline $\begin{array}{l}\text { Zaida Muxí, Shivani Bhardwaj } \\
\text { \& Equal Saree (2010-2013) } \\
\text { (Equal Saree, 2016) }\end{array}$ & $\begin{array}{l}\text { Proyecto Walking India: } \\
\text { Women Resource Zone - } \\
\text { EXPERTOS }\end{array}$ & $\begin{array}{l}\text { Creación de Redes Seguras } \\
\text { y Zonas de Recursos para } \\
\text { mejorar condiciones de vida } \\
\text { para mujeres en la India y en } \\
\text { la ciudad de Barcelona }\end{array}$ & $\begin{array}{l}\text { 1. Seguridad 2. Accesibilidad 3. Equipamiento } \\
\text { urbano } 4 \text {. Servicios básicos } 5 \text {. Escala } \\
\text { peatonal } 6 \text {. Consideración de las principales } \\
\text { actividades de la vida cotidiana de las } \\
\text { mujeres }\end{array}$ \\
\hline $\begin{array}{l}\text { Gender in Mainstreaming Urban } \\
\text { Development. Berlin on the path } \\
\text { towards becoming a metropolis } \\
\text { worth living in for women and men. } \\
\text { (Droste, 2011) }\end{array}$ & $\begin{array}{l}\text { Departamento de Desarrollo } \\
\text { Urbano, Senado de Berlín - } \\
\text { POLÍTICA }\end{array}$ & $\begin{array}{l}\text { Puntos para la planificación } \\
\text { de género }\end{array}$ & $\begin{array}{l}\text { 1. Diversidad y flexibilidad del espacio: } \\
\text { opciones de uso por diferentes grupos } \\
\text { a diferentes horas del día. } 2 \text {. Opciones } \\
\text { de uso social: evitar la perpetuación de } \\
\text { estereotipos de género y roles 3. Espacios } \\
\text { de comunicación e interacción } 4 \text {. Favorecer } \\
\text { la reconciliación del trabajo profesional y } \\
\text { familiar. } 5 \text {. Crear condiciones de seguridad. } \\
6 \text {. Apuntar hacia sustentabilidad y protección } \\
\text { de los recursos naturales. } 7 \text {. Involucramiento } \\
\text { activo y participación. }\end{array}$ \\
\hline
\end{tabular}

Fuente: elaboración propia 
De los casos estudiados y a partir de lo señalado en el apartado anterior se puede concluir que si se busca agregar la perspectiva interseccional a la planeación y al diseño urbano-arquitectónico para favorecer la inclusión y la generación de mejores ciudades y espacios, es fundamental considerar y trabajar en varios puntos, entre los que destacan los que se enlistan en el Cuadro 2.

\section{Cuadro 2.}

\begin{tabular}{|c|c|c|}
\hline 1. Seguridad & $\begin{array}{l}\text { 7. Priorizar al/ la usuario/a humano/a y no humano/a: } \\
\text { reconocimiento de necesidades, actividades/usos y } \\
\text { capacidades }\end{array}$ & 13. Equipamiento urbano \\
\hline 2. Accesibilidad & 8. Flexibilidad espacial & 14. Confort \\
\hline 3. Movilidad & $\begin{array}{l}\text { 9. Nuevas modalidades de ocupación de lo } \\
\text { abandonado o en desuso: la compartida, la temporal, } \\
\text { etcétera }\end{array}$ & $\begin{array}{l}\text { 15. Vitalidad y estímulo a la convivencia de la } \\
\text { diversidad }\end{array}$ \\
\hline 4. Equidad & $\begin{array}{l}\text { 10. Transiciones entre lo privado/individual y lo } \\
\text { público/colectivo }\end{array}$ & $\begin{array}{l}\text { 16. Sustentabilidad, integración de áreas } \\
\text { verdes y de los recursos }\end{array}$ \\
\hline 5. Diversificación de usos, personas y horarios & 11. Diseño a escala humana & $\begin{array}{l}\text { 17. Buscar soluciones a las problemáticas } \\
\text { de los espacios con trabajo colectivo e } \\
\text { integración }\end{array}$ \\
\hline $\begin{array}{l}\text { 6. Desgenerización o disolución de la relación } \\
\text { dicotómica masculino/femenino asociada a los } \\
\text { espacios y a las labores y actividades que se } \\
\text { realizan en ellos }\end{array}$ & 12. Espacios de juego & 18. Fomento económico, educativo y cultural \\
\hline
\end{tabular}

Fuente: elaboración propia

Desde una perspectiva interserseccional, sumar lo colectivo, la diversidad y la equidad a la vida cotidiana es imprescindible para fomentar la inclusión. De ahí que sea necesario incentivar entre los/as habitantes su organización, involucramiento, participación, cooperación, ayuda mutua y responsabilidad compartida, incluyendo la gestión de derechos y deberes a distintas escalas para concebir, mantener y realizar cambios en los espacios del entorno construido. Por lo tanto, no solo es necesario tomar en cuenta los puntos mencionados, también se requiere detectar cuándo se interrelacionan y cómo desde cada ámbito se puede dar respuesta a ellos: ya sea desde la planeación urbana como proceso interdisciplinario o desde el diseño de las características físicas de los lugares como procedimiento que compete principalmente a arquitectos/as y urbanistas.

En síntesis, además de tomar en cuenta todos los planteamientos aquí formulados, efectuar un análisis interseccional urbano-arquitectónico requiere desde un principio reconocer la naturaleza y propiedades de cada sitio, calle, barrio o ciudad dentro de su contexto. Asimismo, hay que contemplar la diversidad de las características físicas, psicológicas y socioculturales de sus moradores -con sus intersecciones- y las actividades que necesitan realizar, así como el carácter de las relaciones sociales existentes. De igual forma, es clave hacerse preguntas acerca de qué problemas de exclusión específicos pueden surgir, especialmente hacia grupos ya vulnerados, y de qué forma pueden ser atendidos desde los procesos de planificación y diseño.

Pese a los avances, con lo aquí expuesto resulta claro que aún falta mucho camino por recorrer en el proceso de integrar la perspectiva de género y la interseccionalidad al quehacer urbano-arquitectónico. Por ello es importante seguir identificando, creando y difundiendo conocimientos y prácticas que incentiven 
la inclusión a nivel general, pero principalmente en el caso de México y América Latina hay que combatir la desigualdad (Falú en Muxí, 2020b) que nos separa reiteradamente y que da pie a la discriminación y a la marginación, por medio de la participación y el diálogo continuo entre la ciudadanía, el gobierno y el sector de producción urbano-arquitectónico. Ya no únicamente es un deseo, sino una absoluta obligación para todos/as los/as que conformamos la sociedad, encontrar maneras de tornar estas fragmentaciones que nos dañan en fusiones que nos beneficien a todos/as.

\section{Figura 3.}

Esbozo de un círculo interseccional urbano-arquitectónico como herramienta para la creación de espacios inclusivos. Podría ser útil encontrar en la zona gris de estas relaciones no solo el encuentro de varias dicotomías, sino el lugar donde se neutraliza su polarización para reflexionarlo y trasladarlo a la realidad de cada caso a estudiar.

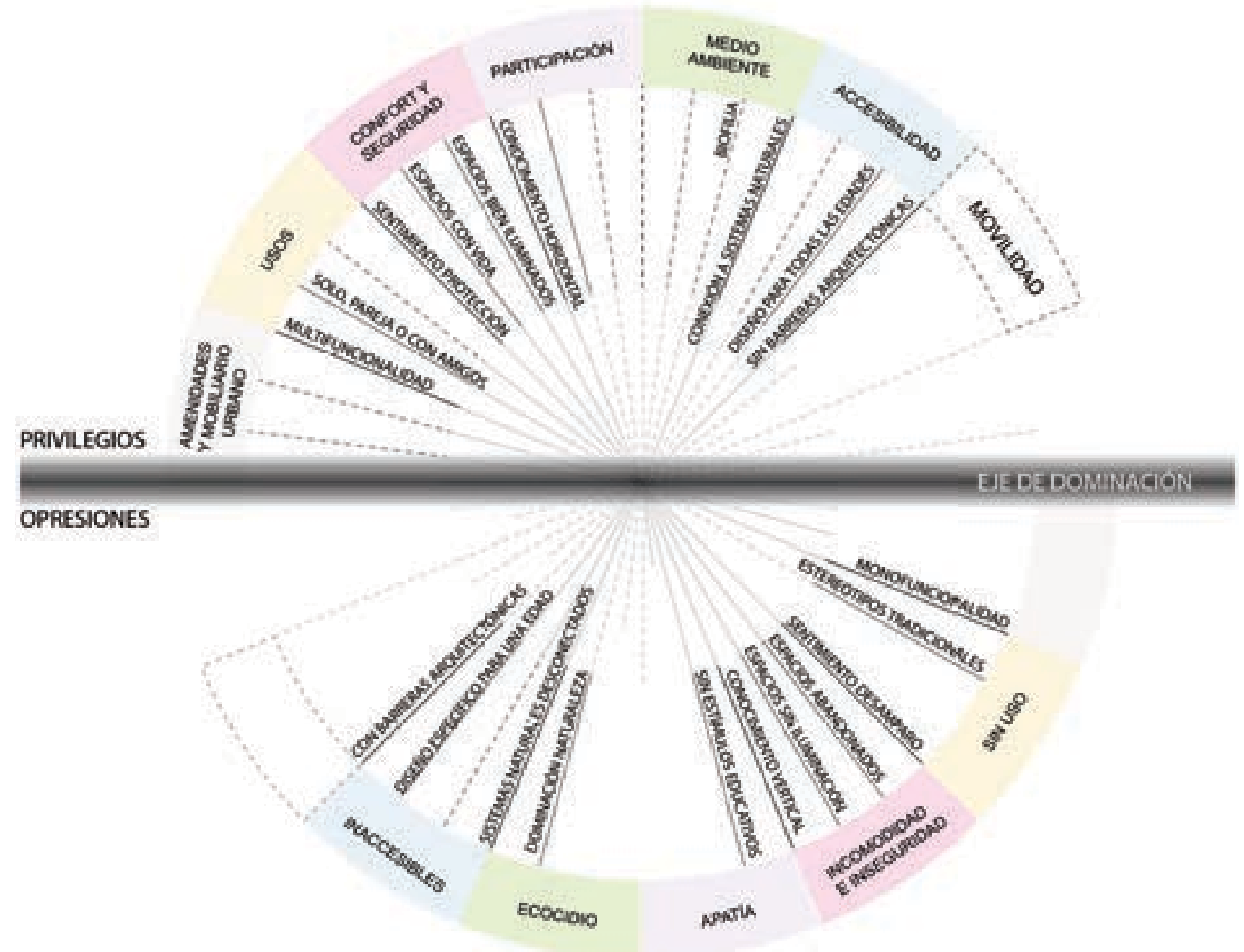

Fuente: Elaboración propia. 


\section{Referencias}

Arellano, M. (2020). Escala humana: sobre resignificación del cuerpo en la producción arquitectónica. Archdaily. Recuperado de https://www.archdaily. $\mathrm{mx} / \mathrm{mx} / 949556 /$ escala-humana-sobre-resignificacion-del-cuerpo-en-la-produccion-arquitectonica

Bagley, Ch. (1974). The built environment as an influence on personality and social behaviour: a spatial study. En D. Canter \& L. Terence, Psychology and the Built Environment (pp. 156-162). USA: Halsted Press.

Colau, A. \& Font, T. (2020). Plenaria 3: Migración y Racismo. III Foro Mundial Ciudades y Territorios de Paz. Ciudad de México, México. Recuperado de https:// www.youtube.com/watch?v=jR9aJkw」_S4\&t=3197s

Collado, S., Staats, H., Corraliza J. A. \& Hartig, T. (2017). Restorative environments and health. En Fleury-Bahi, G., Pol, E. \& Navarro, O., Handbook of environmental psychology and quality of life research (pp. 127-146). Switzerland: Springer.

Collins, P. H. \& Bilge, S. (2016). Intersectionality (Key Concepts). Cambridge: Polity.

Col.lectiu Punt 6. (2016). ¿Qué es el urbanismo feminista? Barcelona. Recuperado de https://www. youtube.com/watch?v=dI4TOCPMMBA\&ab_channel=Col\%C2\%B7lectiuPunt6

Contreras, K. (2020). La significación arquitectónica y urbana en el diseño para propiciar la vinculación espacio-habitante. (Tesis doctoral). Universidad Nacional Autónoma de México, México. 10.13140/ RG.2.2.13833.26721

Crenshaw, K. (2016). The Urgency of Intersectionality. TED talks, USA. Recuperado de https://www. youtube.com/watch?v=ak0e5-UsQ2o\&t=757s

Crawford, M. (2005). Transformations: Women, gender \& psychology. New York: McGraw Hill.
Droste, Ch. (edit.). (2011). Gender in mainstreaming urban development. Berlín: Senatsverwaltung für Stadtentwicklung. Recuperado de http:// www.stadtentwicklung.berlin.de/soziale_stadt/ gender_mainstreaming/download/gender_broschuere_englisch.pdf

Equal Saree. (2016). Walking India. Recuperado de https://equalsaree.org/es/blog/2016/02/24/walking-india/

Etimologías de Chile. (s.a.). Intersección. Recuperado de http://etimologias.dechile.net/?interseccio.n

García, M. L. \& Ramírez, K. M. (2020). Seguridad, espacio público y género: construyendo ciudades seguras para las mujeres y las niñas. Ciudad habitable para todos y todas. México: PUEC UNAM. Recuperado de https://www.youtube.com/watch?v=2vuST3sBfO0

Gehl. (2017). La dimensión humana en el espacio público. Recomendaciones para el análisis y el diseño. Santiago: MINVU, Gobierno de Chile, PNUD.

Gómez-Azpeitia, G., Cruz, S., Gómez, A. \& Alcántara, A. (2005). El entorno arquitectónico como factor asociado a la violencia doméstica en Colima. Iridia, 1(2), 24-35. Recuperado de https://www.researchgate.net/publication/272491463_El_entorno_arquitectonico_como_factor_asociado_a_la_violencia_domestica_en_Colima

Hancock, C. (octubre, 2020). Género y ciudadanía inclusiva: una perspectiva desde Europa. México: UNAM. Conferencia streaming.

La Barbera, M. C. (2016). Interseccionalidad, un concepto viajero: orígenes, desarrollo e implementación en la Unión Europea. Interdisciplina, 4(8), 105-122. http://dx.doi.org/10.22201/ceiich.24485705e.2016.8.5497

Maiztegui, B. (2020). Ciudad del deseo: Escala humana en la ciudad bajo una óptica feminista, trans- 
versal y política. Archdaily. Recuperado de https:// www.archdaily.mx/mx/950347/ciudad-del-deseo-escala-humana-en-la-ciudad-bajo-una-optica-feminista-transversal-y-politica

Muxí, Z. (2020a). Entrevista a Zaida Muxí. Mujer tenía que ser. Intermedio. Entrevista por Sandra Sabatés. La Sexta (televisión), Madrid.

Muxí, Z. (2020b). La producción del hábitat solidario. Seminario sobre el hábitat precario urbano revisado desde la perspectiva de género e interseccionalidad. Chile: SUR Corporación de Estudios Sociales y Educación.
ONU Hábitat. (2017). NAU. Nueva Agenda Urbana. Quito, Ecuador: ONU.

Taylor, R. (2002). Crime prevention through environmental design (CPTED): Yes, No, Maybe, Unknowable, and all of the above. En R. Bechtel et al. (eds), Handbook of environmental psychology (pp. 413-426). New York: Wiley \& S.

UN-HABITAT. (2020). Public space site-specific assessment. Guidelines to achieve quality public spaces at neighbourhood level. Nairobi: UN. Recuperado de https://unhabitat.org/search/go?s=public+space+guidhttps\%3A\%2F\%2Funhabitat. org\%2Fpublic-space-site-specific-assessment-guidelines-to-achieve-quality-public-spaces-atneige

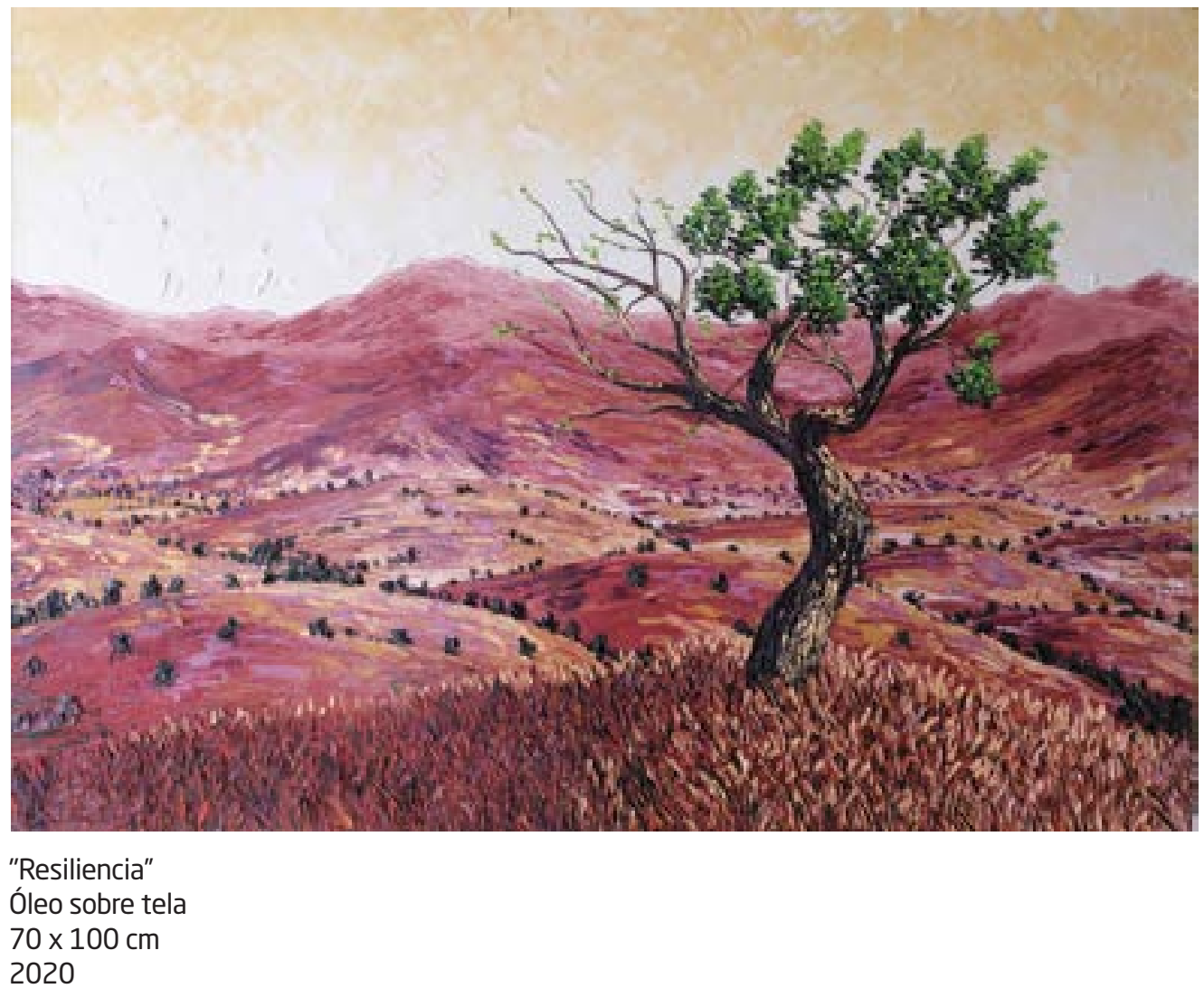

